\title{
Dedekind Sums, Mean Square Value of $L$-Functions at $s=1$ and Upper Bounds on Relative Class Numbers by
}

\author{
Stéphane R. LOUBOUTIN \\ Presented by Jerzy KACZOROWSKI
}

Dedicated to Catherine $H$.

Summary. Explicit formulas for the quadratic mean value at $s=1$ of the Dirichlet $L$-functions associated with the set $X_{f}^{-}$of the $\phi(f) / 2$ odd Dirichlet characters mod $f$ are known. They have been used to obtain explicit upper bounds for relative class numbers of cyclotomic number fields. Here we present a generalization of these results: we show that explicit formulas for quadratic mean values at $s=1$ of Dirichlet $L$-functions associated with subsets of $X_{f}^{-}$can be obtained. As an application we use them to obtain explicit upper bounds for relative class numbers of imaginary subfields of cyclotomic number fields.

1. Introduction. We refer the reader to Was, Chapters 3, 4 and 11] for more background details. Let $f>2$ be an integer. Let $X_{f}$ be the multiplicative group of the $\phi(f)$ Dirichlet characters mod $f$. Let $X_{f}^{-}=\left\{\chi \in X_{f}\right.$; $\chi(-1)=-1\}$ be the set of the $\phi(f) / 2$ odd Dirichlet characters mod $f$. If $H$ is a subgroup of the multiplicative group $(\mathbb{Z} / f \mathbb{Z})^{*}$, we set

$$
X_{f}^{-}(H)=\left\{\chi \in X_{f}^{-} ; \chi_{/ H}=1\right\} .
$$

Let $L(s, \chi)$ be the Dirichlet $L$-function associated with $\chi \in X_{f}$. The mean square value $M(f, H)$ of $L(1, \chi)$ as $\chi$ ranges in $X_{f}^{-}(H)$ is defined by

$$
M(f, H):=\frac{1}{\# X_{f}^{-}(H)} \sum_{\chi \in X_{f}^{-}(H)}|L(1, \chi)|^{2} .
$$

2010 Mathematics Subject Classification: Primary 11R42; Secondary 11M20,11R20,11R29. Key words and phrases: Dirichlet character, $L$-function, mean square value, relative class number, Dedekind sums, cyclotomic field.

Received 29 November 2016.

Published online 14 December 2016. 
We have (see [Lou94, Proposition 1])

$$
L(1, \chi)=\frac{\pi}{2 f} \sum_{a=1}^{f-1} \chi(a) \cot \left(\frac{\pi a}{f}\right) \quad\left(\chi \in X_{f}^{-}\right) .
$$

Let $K$ be an imaginary abelian number field of degree $m=2 n$ and prime conductor $p \geq 3$, i.e. an imaginary subfield of the cyclotomic number field $\mathbb{Q}\left(\zeta_{p}\right)$ (Kronecker-Weber's theorem). The Galois group $\operatorname{Gal}\left(\mathbb{Q}\left(\zeta_{p}\right) / \mathbb{Q}\right)$ is canonically isomorphic to the multiplicative cyclic group $(\mathbb{Z} / p \mathbb{Z})^{*}$, and $H:=$ $\operatorname{Gal}\left(\mathbb{Q}\left(\zeta_{p}\right) / K\right)$ is a subgroup of $(\mathbb{Z} / p \mathbb{Z})^{*}$ of index $m$ and order $(p-1) / m$. Notice that $K$ being imaginary, we have $-1 \notin H$ and the set

$$
X_{K}^{-}:=X_{p}^{-}(H):=\left\{\chi \in X_{p}^{-} ; \chi_{/ H}=1\right\}
$$

is of cardinality $n$. Let $K^{+}$be the maximal real subfield of $K$ of degree $n$ fixed by the complex conjugation. The class number $h_{K^{+}}$of $K^{+}$divides the class number $h_{K}$ of $K$. The relative class number of $K$ is defined by $h_{K}^{-}=h_{K} / h_{K^{+}}$. Let $w_{K}$ be the number of complex roots of unity of $K$. Let $d_{K}$ and $d_{K^{+}}$be the absolute values of the discriminants of $K$ and $K^{+}$. Hence, $d_{K} / d_{K^{+}}=p^{n}$. Using the arithmetic-geometric mean inequality, we have

$$
h_{K}^{-}=\frac{w_{K}}{(2 \pi)^{n}} \sqrt{\frac{d_{K}}{d_{K^{+}}}} \prod_{\chi \in X_{K}^{-}} L(1, \chi) \leq w_{K}\left(\frac{p M(p, H)}{4 \pi^{2}}\right)^{n / 2} .
$$

Up to now this approach has only been used for cyclotomic number fields, i.e. with the choice $H=\{1\}$. Using (2) and the orthogonality relations for characters we obtain (see [Wal])

$$
M(p,\{1\}):=\frac{2}{p-1} \sum_{\chi \in X_{p}^{-}}|L(1, \chi)|^{2}=\frac{\pi^{2}}{6}\left(1-\frac{1}{p}\right)\left(1-\frac{2}{p}\right) \quad(p \geq 3) .
$$

Hence, for $K=\mathbb{Q}\left(\zeta_{p}\right)$ the cyclotomic number field of prime conductor $p \geq 3$ we have

$$
h_{\mathbb{Q}\left(\zeta_{p}\right)}^{-} \leq 2 p\left(\frac{p M(p,\{1\})}{4 \pi^{2}}\right)^{(p-1) / 4} \leq 2 p\left(\frac{p}{24}\right)^{(p-1) / 4}
$$

(see also Lou94, Met).

The aim of the present paper is to develop this approach for proper imaginary subfields $K$ of $\mathbb{Q}\left(\zeta_{p}\right)$ of degree $2 \leq 2 n<p-1$. Since $w_{K}=2$ and the mean square value of $L(1, \chi), \chi \in X_{p}^{-}$, is asymptotic to $\pi^{2} / 6$ by $(4)$, we might expect to have bounds close to

$$
M(p, H) \leq \pi^{2} / 6 \text { and } h_{K}^{-} \leq 2(p / 24)^{n / 2} .
$$

However, it is hopeless to expect such a universal mean square upper bound. Indeed, it is likely that there are infinitely many imaginary abelian number 
fields of a given degree $m=2 n$ and prime conductors $p$ for which

$$
M(p, H)=\frac{1}{n} \sum_{\chi \in X_{K}^{-}}|L(1, \chi)|^{2} \geq\left(\prod_{\chi \in X_{K}^{-}} L(1, \chi)\right)^{2 / n} \gg(\log \log p)^{2}
$$

(see e.g. [CK] and [MW]). Nevertheless, in Theorems 1 and 5, we manage to prove (6) for some non-cyclotomic number fields:

THEOREM 1. Let $p \equiv 1(\bmod 6)$ be a prime integer. Let $K$ be the imaginary subfield $K$ of degree $(p-1) / 3$ of the cyclotomic number field $\mathbb{Q}\left(\zeta_{p}\right)$. Let $H$ be the only subgroup of order 3 of the multiplicative cyclic group $(\mathbb{Z} / p \mathbb{Z})^{*}$. Then $\# X_{p}^{-}(H)=(p-1) / 6$ and we have the mean square value formula (compare with (4))

$$
M(p, H):=\frac{6}{p-1} \sum_{\chi \in X_{p}^{-}(H)}|L(1, \chi)|^{2}=\frac{\pi^{2}}{6}\left(1-\frac{1}{p}\right) .
$$

Hence, the expected bounds (6) hold true, i.e. $M(p, H) \leq \pi^{2} / 6$ and we have the upper bound (compare with (5))

$$
h_{K}^{-} \leq 2(p / 24)^{(p-1) / 6} \text {. }
$$

2. Dedekind and Dedekind-Rademacher sums. For $c \in \mathbb{Z}$ and $d>1$ an integer such that $\operatorname{gcd}(c, d)=1$, the Dedekind sum is defined by

$$
s(c, d)=\frac{1}{4 d} \sum_{n=1}^{d-1} \cot \left(\frac{\pi n}{d}\right) \cot \left(\frac{\pi n c}{d}\right)
$$

(see Apo, Chapter 3, Exercise 11] or [RG, (26)]). It depends only on $c \bmod d$. We also set $s(c, 1)=0$ for $c \in \mathbb{Z}$. Notice that $s(-c, d)=-s(c, d)$ and that $s\left(c^{*}, d\right)=s(c, d)$ whenever $c c^{*} \equiv 1(\bmod d)$ (make the change of variables $n \mapsto n c$ in $\left.s\left(c^{*}, d\right)\right)$. Recall that

$$
s(1, d)=\frac{(d-1)(d-2)}{12 d} \quad(d \geq 1)
$$

(see e.g. [Lou94, Lemma (a)(i)]) and that we have a reciprocity law for Dedekind sums (see e.g. Apo, Theorem 3.7], [RG, (4)] or [Lou15, (7) and (9)])

$$
s(c, d)+s(d, c)=\frac{c^{2}+d^{2}-3 c d+1}{12 c d} \quad(c, d \geq 1, \operatorname{gcd}(c, d)=1) .
$$

For $b, c \in \mathbb{Z}$ and $d>1$ such that $\operatorname{gcd}(b, d)=\operatorname{gcd}(c, d)=1$, the DedekindRademacher sum is defined by

$$
s(b, c, d)=\frac{1}{4 d} \sum_{n=1}^{d-1} \cot \left(\frac{\pi n b}{d}\right) \cot \left(\frac{\pi n c}{d}\right) .
$$


We also set $s(b, c, 1)=0$. Notice that $s(c, d)=s(1, c, d)=s(c, 1, d)$ and that $s(b, c, d)=s(a b, a c, d)$ for any $a \in \mathbb{Z}$ with $\operatorname{gcd}(a, d)=1$. For $b, c, d \geq 1$ and $\operatorname{gcd}(b, c)=\operatorname{gcd}(c, d)=\operatorname{gcd}(d, b)=1$ we have a reciprocity law for Dedekind-Rademacher sums (see e.g. [Rad] or [BR])

$$
s(b, c, d)+s(d, b, c)+s(c, d, b)=\frac{b^{2}+c^{2}+d^{2}-3 b c d}{12 b c d} .
$$

3. A mean square value formula for $L$-functions. We have the following mean square value formula:

$$
M(f,\{1\})=\frac{2}{\phi(f)} \sum_{\chi \in X_{f}^{-}}|L(1, \chi)|^{2}=\frac{\pi^{2}}{6} \frac{\phi(f)}{f}\left(\prod_{p \mid f}\left(1+\frac{1}{p}\right)-\frac{3}{f}\right)
$$

(see [Lou94, Th. 2 and 3], Lou11, Prop. 5], Qi] and [Lou14 for generalizations). Here we prove a general result which when applied with the trivial subgroup $H=\{1\}$ gives $S_{\{1\}}(\delta)=0$ for any divisor $\delta$ of $f$ and 12 :

THEOREM 2. Let $H$ be a subgroup of order $d$ of the multiplicative group $(\mathbb{Z} / f \mathbb{Z})^{*}, f>2$. Assume that $-1 \notin H$, which is the case if $d$ is odd. Set

$$
S_{H}(\delta):=\sum_{1 \neq h \in H} s(h, \delta)
$$

Then $\# X_{f}^{-}(H)=\phi(f) /(2 d)$ and we have the mean square value formula

$$
\begin{aligned}
M(f, H) & :=\frac{2 d}{\phi(f)} \sum_{\chi \in X_{f}^{-}(H)}|L(1, \chi)|^{2} \\
& =\frac{\pi^{2}}{6} \frac{\phi(f)}{f}\left(\prod_{p \mid f}\left(1+\frac{1}{p}\right)-\frac{3}{f}\right)+\frac{2 \pi^{2}}{f} \sum_{\substack{\delta \mid f \\
\delta \neq f}} \frac{\mu(\delta)}{\delta} S_{H}(f / \delta) .
\end{aligned}
$$

Proof. Let $H^{+}=\langle-1, H\rangle$ be the subgroup of order $2 d$ generated by -1 and $H$. For any abelian group $G$ of order $m$ its group of characters $\hat{G}$ is also of order $m$ and

$$
\frac{1}{m} \sum_{\chi \in \hat{G}} \chi(g)= \begin{cases}1 & \text { if } g=1_{G} \\ 0 & \text { otherwise. }\end{cases}
$$

Applying this to the quotient group $G=(\mathbb{Z} / f \mathbb{Z})^{*} / H$ of order $\phi(f) / d$ for which $\hat{G}=\left\{\chi \in X_{f} ; \chi_{/ H}=1\right\}$ and to the quotient group $G^{+}:=$ $(\mathbb{Z} / f \mathbb{Z})^{*} / H^{+}$of order $\phi(f) /(2 d)$ for which $\hat{G}^{+}=\left\{\chi \in X_{f} ; \chi_{/ H^{+}}=1\right\}$, we find that the set $X_{f}^{-}(H)$, which is equal to $\hat{G} \backslash \hat{G}^{+}$, is of cardinality 
$\phi(f) /(2 d)$, and

$$
\frac{2 d}{\phi(f)} \sum_{\chi \in X_{f}^{-}(H)} \chi(x)= \begin{cases}+1 & \text { if } x \in H \\ -1 & \text { if }-x \in H \\ 0 & \text { otherwise }\end{cases}
$$

Hence,

$$
\begin{aligned}
\epsilon(a, b):= & \frac{2 d}{\phi(f)} \sum_{\chi \in X_{f}^{-}(H)} \chi(a) \overline{\chi(b)} \\
& = \begin{cases}+1 & \text { if } \operatorname{gcd}(a, f)=\operatorname{gcd}(b, f)=1 \text { and } a=b h \text { with } h \in H, \\
-1 & \text { if } \operatorname{gcd}(a, f)=\operatorname{gcd}(b, f)=1 \text { and } a=-b h \text { with } h \in H, \\
0 & \text { otherwise. }\end{cases}
\end{aligned}
$$

For $c>1$ and $d \in \mathbb{Z}$ with $\operatorname{gcd}(c, d)=1$, set

$$
\tilde{s}(c, d):=\frac{1}{4 d} \sum_{\substack{n=1 \\ \operatorname{gcd}(n, d)=1}}^{d-1} \cot \left(\frac{\pi n}{d}\right) \cot \left(\frac{\pi n c}{d}\right)=\sum_{\delta \mid d} \frac{\mu(\delta)}{\delta} s(c, d / \delta),
$$

by the Möbius inversion formula and the convention that $s(c, 1)=0$ for $c \in \mathbb{Z}$. Using (2), we obtain

$$
\begin{aligned}
M(f, H) & =\frac{\pi^{2}}{4 f^{2}} \sum_{a=1}^{f-1} \sum_{b=1}^{f-1} \epsilon(a, b) \cot \left(\frac{\pi a}{f}\right) \cot \left(\frac{\pi b}{f}\right) \\
& =\frac{\pi^{2}}{2 f^{2}} \sum_{h \in H} \sum_{\substack{b=1 \\
\operatorname{gcd}(b, f)=1}}^{f-1} \cot \left(\frac{\pi b}{f}\right) \cot \left(\frac{\pi h b}{f}\right) \\
& =\frac{2 \pi^{2}}{f} \sum_{h \in H} \tilde{s}(h, f) \\
& =\frac{2 \pi^{2}}{f} \sum_{h \in H} \sum_{\delta \mid f} \frac{\mu(\delta)}{\delta} s(h, f / \delta) \\
& =\frac{2 \pi^{2}}{f} \sum_{\delta \mid f} \frac{\mu(\delta)}{\delta} s(1, f / \delta)+\frac{2 \pi^{2}}{f} \sum_{1 \neq h \in H} \sum_{\delta \mid f} \frac{\mu(\delta)}{\delta} s(h, f / \delta) .
\end{aligned}
$$

Since $s(h, 1)=0$ for any $h$, we can restrict $\delta \mid f$ to $f \neq \delta \mid f$ in the second term of the last line. By $(9)$ we have $s(1, d)=(d-3+2 / d) / 12$ for $d \geq 1$ and

$$
\frac{2 \pi^{2}}{f} \sum_{\delta \mid f} \frac{\mu(\delta)}{\delta} s(1, f / \delta)=\frac{\pi^{2}}{6}\left(\sum_{\delta \mid f} \frac{\mu(\delta)}{\delta^{2}}-\frac{3}{f} \sum_{\delta \mid f} \frac{\mu(\delta)}{\delta}+\frac{2}{f^{2}} \sum_{\delta \mid f} \mu(\delta)\right) .
$$


Finally, since $\sum_{\delta \mid f} \mu(\delta) / \delta^{2}=\prod_{p \mid f}\left(1-1 / p^{2}\right), \sum_{\delta \mid f} \mu(\delta) / \delta=\prod_{p \mid f}(1-1 / p)$ and $\sum_{\delta \mid f} \mu(\delta)=0$, the desired result follows.

Corollary 3. Let $d$ be an odd divisor of $p-1$, where $p \geq 3$ is an odd prime number. Let $H=\left\langle h_{0}\right\rangle$ be the only cyclic subgroup of $(\mathbb{Z} / p \mathbb{Z})^{*}$ of order $d$, where $h_{0}$ is any element of order $d$ in the multiplicative cyclic group $(\mathbb{Z} / p \mathbb{Z})^{*}$. Then $\# X_{p}^{-}(H)=(p-1) /(2 d)$ and we have the mean square value formula

$$
\begin{aligned}
M(p, H) & :=\frac{2 d}{p-1} \sum_{\chi \in X_{p}^{-}(H)}|L(1, \chi)|^{2} \\
& =\frac{\pi^{2}}{6}\left(1+\frac{24 \sum_{k=1}^{(d-1) / 2} s\left(h_{0}^{k}, p\right)-3}{p}+\frac{2}{p^{2}}\right) .
\end{aligned}
$$

Proof. Notice that $\delta=1$ is the only $\delta \neq p$ dividing $p$, and

$$
S_{H}(p)=\sum_{1 \neq h \in H} s(h, p)=\sum_{k=1}^{d-1} s\left(h_{0}^{k}, p\right)=2 \sum_{k=1}^{(d-1) / 2} s\left(h_{0}^{k}, p\right),
$$

using $h_{0}^{d-k}=h_{0}^{-k}$ and $s\left(c^{*}, p\right)=s(c, p)$ whenever $c c^{*} \equiv 1(\bmod p)$.

4. Proof of Theorem 1. Let $h_{0}$ of order 3 be a generator of $H$. Corollary 3 applied with $d=3$ gives

$$
M(p, H)=\frac{\pi^{2}}{6}\left(1+\frac{24 s\left(h_{0}, p\right)-3}{p}+\frac{2}{p^{2}}\right) .
$$

Then the lemma below gives the desired result (we thank Abdelmejid Bayad for having sent us its present proof using Dedekind-Rademacher sums):

Lemma 4. Assume that $f=a^{2}+a b+b^{2}>3$, where $a \in \mathbb{Z}, b \geq 1$ and $\operatorname{gcd}(a, b)=1$. Then $\operatorname{gcd}(a, f)=\operatorname{gcd}(b, f)=1, h_{0}=a / b$ is of order 3 in the multiplicative group $(\mathbb{Z} / f \mathbb{Z})^{*}$ and

$$
s\left(h_{0}, f\right)=s\left(h_{0}^{2}, f\right)=\frac{f-1}{12 f} .
$$

Consequently, if $p \equiv 1(\bmod 6)$ is a prime integer then for any $h \in(\mathbb{Z} / p \mathbb{Z})^{*}$ of order 3 in this group we have $s(h, p)=\frac{p-1}{12 p}$.

Proof. Assume that $a \geq 1$. Then $f \equiv b^{2}(\bmod a)$ yields $s(b, f, a)=$ $s\left(b, b^{2}, a\right)=s(1, b, a)=s(b, a)$, and $f \equiv a^{2}(\bmod b)$ gives $s(f, a, b)=$ $s\left(a^{2}, a, b\right)=s(a, 1, b)=s(a, b)$. The reciprocity law for Dedekind sums yields

$$
s(b, f, a)+s(f, a, b)=s(b, a)+s(a, b)=\frac{a^{2}+b^{2}-3 a b+1}{12 a b}
$$


and the reciprocity for the Dedekind-Rademacher sums implies

$$
s(a, b, f)+s(b, f, a)+s(f, a, b)=\frac{a^{2}+b^{2}+f^{2}-3 a b f}{12 a b f} .
$$

Hence, we finally obtain

$$
\begin{aligned}
s\left(h_{0}, f\right) & =s(a, b, f)=\frac{a^{2}+b^{2}+f^{2}-3 a b f}{12 a b f}-\frac{a^{2}+b^{2}-3 a b+1}{12 a b} \\
& =\frac{f-1}{12 f} .
\end{aligned}
$$

Now, assume that $a \leq-1$. Then

$$
s(b, f,-a)+s(f,-a, b)=s(b,-a)+s(-a, b)=\frac{a^{2}+b^{2}+3 a b+1}{-12 a b}
$$

and

$$
s(-a, b, f)+s(b, f,-a)+s(f,-a, b)=\frac{a^{2}+b^{2}+f^{2}+3 a b f}{-12 a b f} .
$$

Hence,

$$
s(a, b, f)=-s(-a, b, f)=-\left(\frac{a^{2}+b^{2}+f^{2}+3 a b f}{-12 a b f}-\frac{a^{2}+b^{2}+3 a b+1}{-12 a b}\right)
$$

and $s\left(h_{0}, f\right)=s(a, b, f)=\frac{f-1}{12 f}$.

Since $h_{0}^{2}=h_{0}^{*}$, i.e. $h_{0}^{2} h_{0}=h_{0}^{3} \equiv 1(\bmod f)$, we have $s\left(h_{0}^{2}, f\right)=s\left(h_{0}, f\right)$.

Finally, let $p \equiv 1(\bmod 6)$ be a prime integer. Then $p$ splits completely in the imaginary quadratic number field $L=\mathbb{Q}(\sqrt{-3})$. Since the class number of $L$ is equal to 1 , there is an algebraic integer $\alpha=a+b(1+\sqrt{-3}) / 2$ in $L$, $a, b \in \mathbb{Z}$, such that $p=N_{L / \mathbb{Q}}(\alpha)=a^{2}+a b+b^{2}$. Clearly, $\operatorname{gcd}(a, b)=1$ and we may assume that $b \geq 1$. Then $h_{0}=a / b$ and $h_{0}^{2}$ are the only elements of order 3 in the multiplicative cyclic group $(\mathbb{Z} / p \mathbb{Z})^{*}$.

5. Some imaginary abelian number fields of conductor $p \equiv 1$ $(\bmod 5)$ and degree $(p-1) / 5$. We conclude this paper by explaining why it is probably hopeless to find a statement as neat as Theorem 1 for subgroups $H$ of $(\mathbb{Z} / p \mathbb{Z})^{*}$ of odd order $d$ greater than 3 . Even for $d=5$ and $p \equiv 1(\bmod 10)$ we could not find a generalization of Theorem 1, i.e. a formula for $M(p, H)$, where $H$ is the only subgroup of order 5 of the multiplicative group $(\mathbb{Z} / p \mathbb{Z})^{*}$. These prime integers $p \equiv 1(\bmod 10)$ are the prime integers that split completely in the cyclotomic number field $\mathbb{Q}\left(\zeta_{5}\right)$ of class number one, therefore they are the prime integers $p \neq 5$ of the form

$$
p=P(a, b, c, d):=N_{\mathbb{Q}\left(\zeta_{5}\right) / \mathbb{Q}}\left(a-b \zeta_{5}-c \zeta_{5}^{2}-d \zeta_{5}^{3}\right), \quad a, b, c, d \in \mathbb{Z} .
$$


The first problem is that we could not find a closed formula for an element $h_{0}$ of order 5 . Even for the prime integers $p \neq 5$ of the form

$$
p=N_{\mathbb{Q}\left(\zeta_{5}\right) / \mathbb{Q}}\left(a-b \zeta_{5}\right)=a^{4}+a^{3} b+a^{2} b^{2}+a b^{3}+b^{4},
$$

for which $h_{0}=a / b$ is of order 5 in the multiplicative group $(\mathbb{Z} / p \mathbb{Z})^{*}$, we could not find a generalization of Lemma 4 , i.e. closed formulas for $s(a, b, f)$ and $s\left(a^{2}, b^{2}, f\right)$. At least, for $b=1$, i.e. for the prime integers $p \neq 5$ of the form $p=N_{\mathbb{Q}\left(\zeta_{5}\right) / \mathbb{Q}}\left(a-\zeta_{5}\right)=a^{4}+a^{3}+a^{2}+a+1$ we have:

THEOREM 5. Let $p \neq 5$ be a prime of the form

$$
p=a^{4}+a^{3}+a^{2}+a+1,
$$

e.g. $(a, p) \in\{(-2,11),(2,31),(-3,61),(-5,521),(7,2801),(-10,9091)\}$. Then $p \equiv 1(\bmod 10)$. Let $H$ be the only subgroup of order 5 of the multiplicative cyclic group $(\mathbb{Z} / p \mathbb{Z})^{*}$ and let $K$ be the imaginary subfield of degree $(p-1) / 5$ of the cyclotomic number field $\mathbb{Q}\left(\zeta_{p}\right)$. Then $\# X_{p}^{-}(H)=(p-1) / 10$ and we have the mean square value formula (compare with (4) and (7))

$$
M(p, H):=\frac{10}{p-1} \sum_{\chi \in X_{p}^{-}(H)}|L(1, \chi)|^{2}=\frac{\pi^{2}}{6}\left(1+\frac{2 a(a+1)^{2}-1}{p}\right) .
$$

Hence, for $a \leq-2$ the expected bounds (6) hold true, i.e. $M(p, H) \leq \pi^{2} / 6$ and (compare with (5) and (8)) $h_{K}^{-} \leq 2(p / 24)^{(p-1) / 10}$.

Proof. Applying Corollary 3 with $d=5$ and $h_{0}=a$, we have

$$
M(p, H)=\frac{\pi^{2}}{6}\left(1+\frac{24\left(s(a, p)+s\left(a^{2}, p\right)\right)-3}{p}+\frac{2}{p^{2}}\right) .
$$

By Lemma 6, the desired result follows.

Lemma 6. Let $q \geq 3$ be an odd prime. For $a \in \mathbb{Z}, a \neq-1,0,1$, set $f:=\left(a^{q}-1\right) /(a-1)=a^{q-1}+a^{q-2}+\cdots+a+1>1$, an odd integer. Assume that $f \neq q$. Then $a$ is of order $q$ in the multiplicative group $(\mathbb{Z} / f \mathbb{Z})^{*}$, and

$$
s(a, f)=\frac{(f-1)\left(f-a^{2}-1\right)}{12 a f} \quad \text { and } \quad s\left(a^{2}, f\right)=\frac{(f-1)^{2}+a^{2}\left(a^{2}-f\right)}{12 a^{2} f} .
$$

Proof. Using (10) we get

$$
s(a, f)=\frac{a^{2}+f^{2}-3 a f+1}{12 a f}-s(f, a) .
$$

Using $f \equiv 1(\bmod a)$ and $(9)$ we get $s(f, a)=s(1, a)=(a-1)(a-2) /(12 a)$ The desired result for $s(a, f)$ follows.

In the same way, $a^{2} \equiv 1(\bmod a+1)$ implies

$$
s\left(a^{2}, a+1\right)=s(1, a+1)=\frac{a(a-1)}{12(a+1)},
$$


and $f \equiv a+1\left(\bmod a^{2}\right)$ and 10$)$ yield

$$
\begin{gathered}
s\left(f, a^{2}\right)=s\left(a+1, a^{2}\right)=\frac{(a+1)^{2}+a^{4}-3(a+1) a^{2}+1}{12(a+1) a^{2}}-s\left(a^{2}, a+1\right), \\
s\left(f, a^{2}\right)=-\left(a^{2}-1\right) /\left(6 a^{2}\right) \text { and } \\
s\left(a^{2}, f\right)=\frac{a^{4}+f^{2}-3 a^{2} f+1}{12 a^{2} f}-s\left(f, a^{2}\right) .
\end{gathered}
$$

The desired result for $s\left(a^{2}, f\right)$ follows.

\section{Conclusion: two open problems}

1. For the cyclotomic fields of prime conductors we have the following improvement on (5):

$$
h_{\mathbb{Q}\left(\zeta_{p}\right)}^{-} \leq 2 p(p / 32)^{(p-1) / 4}
$$

(see [Feng and [Lou11]). It would be worth proving a similar improvement on (8), i.e. proving that if $K$ is an imaginary subfield of degree $(p-1) / 3$ of the cyclotomic number field $\mathbb{Q}\left(\zeta_{p}\right)$, where $p \equiv 1(\bmod 6)$, then

$$
h_{K}^{-} \leq 2(p / 32)^{(p-1) / 6} \text {. }
$$

2. As explained in Section 5, it seems hopeless for a given odd $d>3$ to obtain an explicit formula for $M(p, H)$ that woull hold true for all the prime integers $p \equiv 1(\bmod 2 d)$, where $H$ is the only subgroup of order $d$ of the group $(\mathbb{Z} / p \mathbb{Z})^{*}$. Nevertheless, it would be worth obtaining an asymptotic $M(p, H)=\left(\pi^{2} / 6\right)(1+o(1))$.

\section{References}

[Apo] M. T. Apostol, Modular Functions and Dirichlet Series in Number Theory, Grad. Texts in Math. 41, Springer, New York, 1976.

[BR] A. Bayad and A. Raouj, Reciprocity formulae for multiple Dedekind-Rademacher sums, C. R. Math. Acad. Sci. Paris 349 (2011), 131-136.

[CK] P. J. Cho and A. H. H. Kim, Dihedral and cyclic extensions with large class numbers, J. Théor. Nombres Bordeaux 24 (2012), 583-603.

[Feng] K. Q. Feng, On the first factor of the class number of a cyclotomic field, Proc. Amer. Math. Soc. 84 (1974), 479-482.

[Lou94] S. Louboutin, Quelques formules exactes pour des moyennes de fonctions $L$ de Dirichlet, Canad. Math. Bull. 36 (1993), 190-196; Addendum, Canad. Math. Bull. 37 (1994), 89.

[Lou11] S. Louboutin, Mean values of L-functions and relative class numbers of cyclotomic fields, Publ. Math. Debrecen 78 (2011), 647-658.

[Lou14] S. Louboutin, A twisted quadratic moment for Dirichlet L-functions, Proc. Amer. Math. Soc. 142 (2014), 1539-1544.

[Lou15] S. Louboutin, Twisted quadratic moments for Dirichlet L-functions, Bull. Korean Math. Soc. 52 (2015), 2095-2105. 
[Met] T. Metsänkylä, Class numbers and $\mu$-invariants of cyclotomic fields, Proc. Amer. Math. Soc. 43 (1974), 299-300.

[MW] H. L. Montgomery and P. J. Weinberger, Real quadratic fields with large class number, Math. Ann. 225 (1977), 173-176.

[Qi] M.-G. Qi, A class of mean square formulas for L-functions, J. Tsinghua Univ. 31 (1991), no. 3, 34-41 (in Chinese).

[Rad] H. Rademacher, Generalization of the reciprocity formula for Dedekind sums, Duke Math. J. 21 (1954), 391-397.

[RG] H. Rademacher and E. Grosswald, Dedekind Sums, Carus Math. Monogr. 16, Math. Assoc. Amer., Washington, DC, 1972.

[Wal] H. Walum, An exact formula for an average of $L$-series, Illinois J. Math. 26 (1982), 1-3.

[Was] L. C. Washington, Introduction to Cyclotomic Fields, 2nd ed., Grad. Texts in Math. 83, Springer, New York, 1997.

Stéphane R. Louboutin

Aix Marseille Université

CNRS, Centrale Marseille, I2M

Marseille, France

E-mail: stephane.louboutin@univ-amu.fr 